\title{
Is HCG 31 undergoing a merger or a fly-by interaction?
}

\author{
M. G. Richer ${ }^{1}$, L. Georgiev², M. Rosado ${ }^{2}$, A. Bullejos ${ }^{3,2}$, M. Valdez-Gutiérrez ${ }^{4,5}$, and D. Dultzin-Hacyan ${ }^{2}$ \\ 1 Observatorio Astronómico Nacional, Instituto de Astronomía, UNAM, PO Box 439027, San Diego, CA 92143-9027, USA \\ 2 Instituto de Astronomía, UNAM, Apartado Postal 70-264, Ciudad Universitaria, 04510 México, D.F., México \\ e-mail: [georgiev, margarit, deborah]@astroscu.unam.mx \\ 3 Instituto de Astrofísica de Canarias, Vía Láctea, 38200 La Laguna, Tenerife, Spain \\ e-mail: almudena@astroscu.unam.mx \\ ${ }^{4}$ LERMA, Observatoire de Paris, 61 Av. de l'Observatoire, 75014 Paris, France \\ e-mail: margarita.valdez@obspm.fr \\ 5 Instituto Nacional de Astrofísica, Óptica y Electrónica, Apartado Postal 51, 72000 Puebla, Puebla, México \\ e-mail: mago@inaoep.mx
}

Received 21 August 2001 / Accepted 23 September 2002

\begin{abstract}
We present Fabry-Perot and multi-object spectroscopy of the galaxies in Hickson compact group 31 (HCG 31). Based upon our $\mathrm{H} \alpha$ data cubes, galaxies $\mathrm{A}$ and $\mathrm{C}$ are a single entity, showing no discontinuity in their kinematics. Kinematically, galaxy $\mathrm{E}$ is probably a component of the $\mathrm{A}+\mathrm{C}$ complex; otherwise it is a recently detached fragment. Galaxy $\mathrm{F}$ appears, both kinematically and chemically, to have formed from material tidally removed from the A+C complex. Galaxies B and $G$ are kinematically distinct from this complex. Galaxy Q also has a radial velocity compatible with group membership. Galaxies $\mathrm{A}, \mathrm{B}, \mathrm{C}$, and $\mathrm{F}$ have nearly identical oxygen abundances, despite spanning a luminosity range of 5 mag. Galaxy B's oxygen abundance is normal for its luminosity, while galaxy F's abundance is that expected given its origin as a tidal fragment of the $\mathrm{A}+\mathrm{C}$ complex. The oxygen abundances in galaxies $\mathrm{A}$ and $\mathrm{C}$ are also understandable if the $\mathrm{A}+\mathrm{C}$ complex is a late-type spiral suffering strong gas inflow and star formation as a result of a tidal interaction. Given the kinematics of both the galaxies and the $\mathrm{H}_{\mathrm{I}}$ gas, the oxygen abundances, and the position of galaxy $\mathrm{G}$, we propose that an interaction of galaxy $\mathrm{G}$ with the $\mathrm{A}+\mathrm{C}$ complex, rather than a merger of galaxies $\mathrm{A}$ and $\mathrm{C}$, is a more complete explanation for the tidal features and other properties of HCG 31. In this case, the A+C complex need not be a merger in progress, though this is not ruled out.
\end{abstract}

Key words. galaxies: abundances - galaxies: evolution - galaxies: formation - galaxies: individual: NGC 1741 galaxies: clusters: individual: HCG 31

\section{Introduction}

The Hickson compact group of galaxies 31 (HCG 31; Hickson 1982) has recently proven an interesting laboratory for studying various issues related to galaxy formation and merging. HCG 31 is physically compact. Six of its members fall within the dimensions of a typical giant galaxy, share a common H I envelope (Williams et al. 1991), and span a small range in radial velocities (Rubin et al. 1990; Hickson et al. 1992). It is therefore no surprise that HCG 31 shows a wide array of evidence for galaxy interaction including: tidal tails and irregular morphology, complex kinematics, and vigourous star formation, including starbursts with Wolf-Rayet spectral features (Rubin et al. 1990; Iglesias-Páramo \& Vílchez 1997; Izotov \& Thuan 1998; Johnson et al. 1999; Johnson \& Conti 2000).

Rubin et al. (1990) conclude that HCG 31 will appear as a single normal elliptical galaxy within a few orbital periods. Here, we differ somewhat from this conclusion. Although we find abundant kinematical evidence for recent gravitational interaction among the group members, we suggest that there are

Send offprint requests to: M. G. Richer,

e-mail: richer@astrosen.unam.mx other explanations besides imminent coalescence. Likewise, we find chemical abundances for the different components that are unlike those of normal dwarf irregulars, but again argue that their origins may be easily understood. Given the abundant evidence for gravitational interaction, a merger may yet occur in HCG 31, but we find that it need not be currently underway.

In the following section, we present our data and their reduction. In Sect. 3, we present our results. In Sect. 4, we discuss these results and their implications for the state of HCG 31 . In Sect. 5, we present our interpretation of the past and future evolution of HCG 31. In Sect. 6, we summarize our conclusions. Throughout, we adopt the galaxy nomenclature of Rubin et al. (1990) and we assume a distance of 58 Mpc for HCG 31, based upon a mean redshift of 0.0137 (Hickson et al. 1992) and a Hubble constant of $71 \mathrm{~km} \mathrm{~s}^{-1} \mathrm{Mpc}^{-1}$ (Mould et al. 2000). A preliminary version of some of our results was presented by Rosado et al. (1999).

\section{Observations and reductions}

The Fabry-Perot (FP) observations of HCG 31 were carried out on 1 November 1997 at the f/7.5 Cassegrain focus 


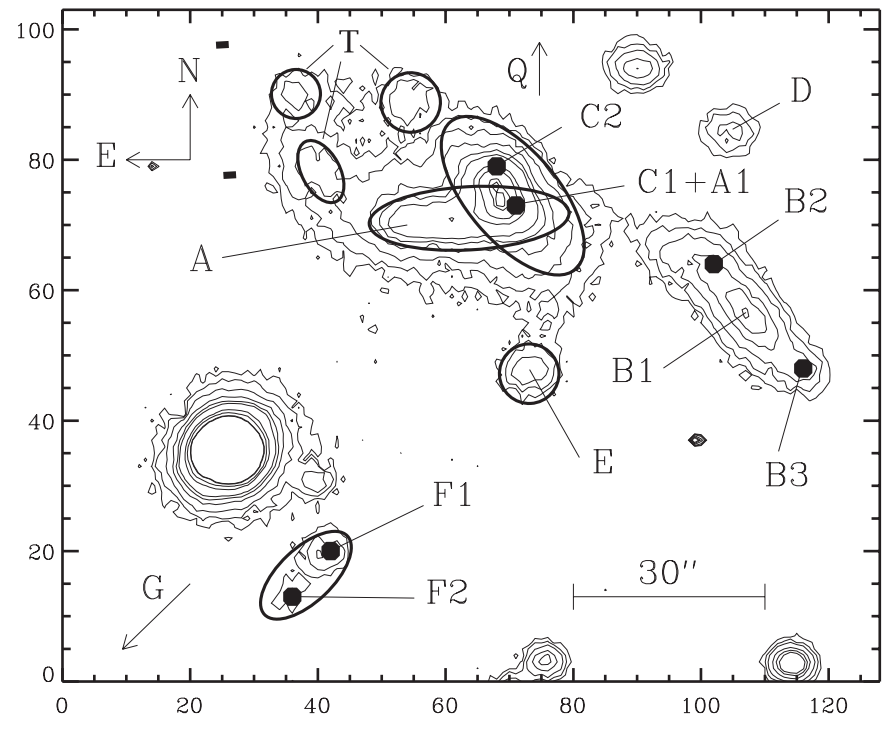

Fig. 1. The field of HCG 31 in the $V$-band. Galaxies A, B, C, D, E, and $\mathrm{F}$ are shown; galaxies $\mathrm{G}$ and $\mathrm{Q}$ are to the south-east and north, respectively, as indicated by the arrows. Deeper, higher-resolution images may be found in Rubin et al. (1990), Iglesias-Páramo \& Vílchez (1997), Johnson et al. (1999), and Johnson \& Conti (2000). The ellipses are meant to illustrate schematically the positions of galaxies A, C, E, F and the tidal dwarf candidates (denoted by "T"; Hunsberger et al. 1996). Objects within the component galaxies are labelled using the galaxy component letter and a sequence number, e.g., F1 and F2 are subcomponents of galaxy F. Where sub-components are labelled, the parent galaxies are not, to reduce the clutter. North is up and east is to the left, as indicated, and $1^{\prime \prime}=281 \mathrm{pc}$. Unlabelled objects are foreground stars, except that north-east of galaxy D, which is a reflection. The filled circles indicate the locations of the apertures used for multi-object spectroscopy.

of the $2.1 \mathrm{~m}$ telescope of the Observatorio Astronómico Nacional in San Pedro Mártir, B.C., México using the UNAM Scanning Fabry-Perot Interferometer PUMA (Rosado et al. 1995). The scanning FP interferometer is based upon an ET-50 Queensgate Instruments etalon with a servo-stabilization system. The $\mathrm{H} \alpha$ line is observed in interference order 330, within a free spectral range of $19.89 \AA$, and sampled at 48 steps of $0.43 \AA$ separation $\left(18.9 \mathrm{~km} \mathrm{~s}^{-1}\right)$. The spectral resolution of these observations is $38.4 \mathrm{~km} \mathrm{~s}^{-1}$. The effective finesse obtained with this setup was 24 . Since HCG 31 subtends only $2^{\prime}$ and we were careful to place it at the center of the instrument's field of view $\left(10^{\prime}\right)$ when acquiring the data cubes, the effective finesse of the observations is the same. In any case, PUMA does not show important variations of the effective finesse across the field. A $30 \AA$ interference filter, centered at the wavelength of redshifted $\mathrm{H} \alpha$, was used to isolate the $\mathrm{H} \alpha$ emission line from HCG 31. The detector was a $1024 \times 1024$, thinned Tektronix CCD. The image scale was 0.59 pixel $^{-1}$, yielding a $10^{\prime}$ field of view. In order to increase the $S / N$ of the observations, the detector was used with a $2 \times 2$ pixel binning.

We obtained two data cubes of HCG 31 in $\mathrm{H} \alpha$ with exposure times of $48 \mathrm{~min}$ each $(1 \mathrm{~min} / \mathrm{channel})$. These data cubes were co-added to enhance the $S / N$ of the faint regions. The seeing was about 1 '.2. The transparency conditions were rather good and we obtained both cubes during dark time.
Nevertheless, we corrected both data cubes for transparency variations before co-adding them. This was done using two field stars (located outside the region shown in Fig. 1) and we verified that the profiles were similar.

We obtained wavelength calibration data cubes of a $\mathrm{Ne}$ lamp at the beginning and end of the observations, which also serve to check for possible equipment flexures. Since the redshifted $\mathrm{H} \alpha$ emission of HCG 31 differs from the wavelength of the calibration lamp, a phase shift correction was applied, which amounted to a shift in the zero-point of the velocities of $22 \mathrm{~km} \mathrm{~s}^{-1}$. The CIGALE software package (Le Coarer et al. 1993) was used to apply this phase shift correction, to remove cosmic rays, calibrate in wavelength, and construct the radial velocity cubes. We also used some routines from the Image Reduction and Analysis Facility (IRAF) ${ }^{1}$ for parts of the data reduction.

The multi-object spectroscopy of the galaxies in HCG 31 was obtained with the $2.1 \mathrm{~m}$ telescope of the Observatorio Astrofísico Guillermo Haro in Cananea, México on 10 January 1999. The LFOSC spectrograph was used, which is a transmission spectrograph employing a grism as the dispersing element (Zickgraf et al. 1997). The spectrograph's dispersion was approximately $5.5 \AA /$ pix and the spectra typically spanned from $4000 \AA$ to $6600 \AA$, though the exact spectral range varied according to the object's position within the spectrograph's field of view. The detector was a $576 \times 384$ EEV CCD. Since the spectrograph has very poor response in the blue, no order-sorting filter was used. The objects were selected for spectroscopy using focal plane masks made from previouslyacquired images. The only restriction for object selection is that they may not be aligned in declination since the dispersion axis is oriented east-west. Pairs of holes were cut for object and sky at the same right ascension so as to ensure identical spectral coverage. The holes were $3^{\prime \prime}$ in diameter.

The objects selected for spectroscopy are shown in Fig. 1, with the exception of galaxy Q (Rubin et al. 1990), which is to the north of the field. The total integration time was 1.5 hours.

The standard stars HD 19445, HD 74721, and HD 109995 were observed for flux calibration. The standard stars were observed through masks cut for various object fields.

The multi-object spectroscopy was reduced using the IRAF software package, specifically the noao.imred.specred package. The bias images were first combined and the result subtracted from all of the images. Pixel-to-pixel variations were then removed using spectra of the internal lamp. Subsequently, the sky was subtracted from each object. Spectra of the Ne-Ar lamp were used for wavelength calibration. Finally, the flux calibration was made using the standard star observations.

\section{Results}

Figures 2 and 3 present the radial velocity and velocity dispersion maps resulting from our Fabry-Perot spectroscopy at $\mathrm{H} \alpha$.

\footnotetext{
${ }^{1}$ IRAF is distributed by the National Optical Astronomical Observatories, which is operated by the Associated Universities for Research in Astronomy, Inc., under contract to the National Science Foundation.
} 




Fig. 2. The velocity map of HCG 31 in $\mathrm{H} \alpha$. The velocities are heliocentric velocities. Panel a) shows the velocities for galaxies A, C, E, and F; panel b) shows the velocities for galaxy B; and panel c) shows the velocities for galaxy G. In all cases, the colour bars show the total range of radial velocities. The ellipses are again meant to illustrate the positions of galaxies A, C, E, F, and the tidal candidates, as in Fig. 1. In general, both our radial velocities and velocity profiles are in good agreement with those of Rubin et al. (1990). The continuity of the kinematics for galaxies $\mathrm{A}, \mathrm{C}$, and $\mathrm{E}$ is particularly striking, and probably indicates that these three components are a single entity. Galaxy Q was not detected in our Fabry-Perot data cubes, while the $\mathrm{H} \alpha$ line from galaxy D is shifted outside our filter by this galaxy's much higher radial velocity (Hickson et al. 1992). In all panels, north is up, east is to the left, and $1^{\prime \prime}=281 \mathrm{pc}$.

To compute these maps, we computed the average intensity and its standard deviation over all channels at each spatial pixel. Then, we determined the median value of this standard deviation over all spatial pixels in the image and adopted this as representative of the noise in the sky throughout the image. Finally, at each spatial pixel, the maximum of the intensities in all channels was compared to this noise value. For those spatial pixels where the maximum intensity in some channel exceeded five times the noise value, a Gaussian was fitted to the velocity profile. The peak position and the standard deviation of this Gaussian fit were adopted as the radial velocity and velocity dispersion, respectively, at that spatial position.

In Fig. 2, our radial velocities and velocity profiles are in good agreement with those found by Rubin et al. (1990). In Fig. 4, we present several simulated profiles for the positions and position angles corresponding to Figs. 2 and 3 of Rubin et al. (1990). Galaxy Q was not detected in our data cubes, while galaxy D's much higher radial velocity shifted its $\mathrm{H} \alpha$ line outside our filter.

In Fig. 3, we present the velocity dispersions in galaxies $\mathrm{A}$ and $\mathrm{C}$. We present a map of the velocity dispersion for galaxies $\mathrm{A}$ and $\mathrm{C}$ only, since no other galaxies showed significant internal variations in velocity dispersion. The velocity

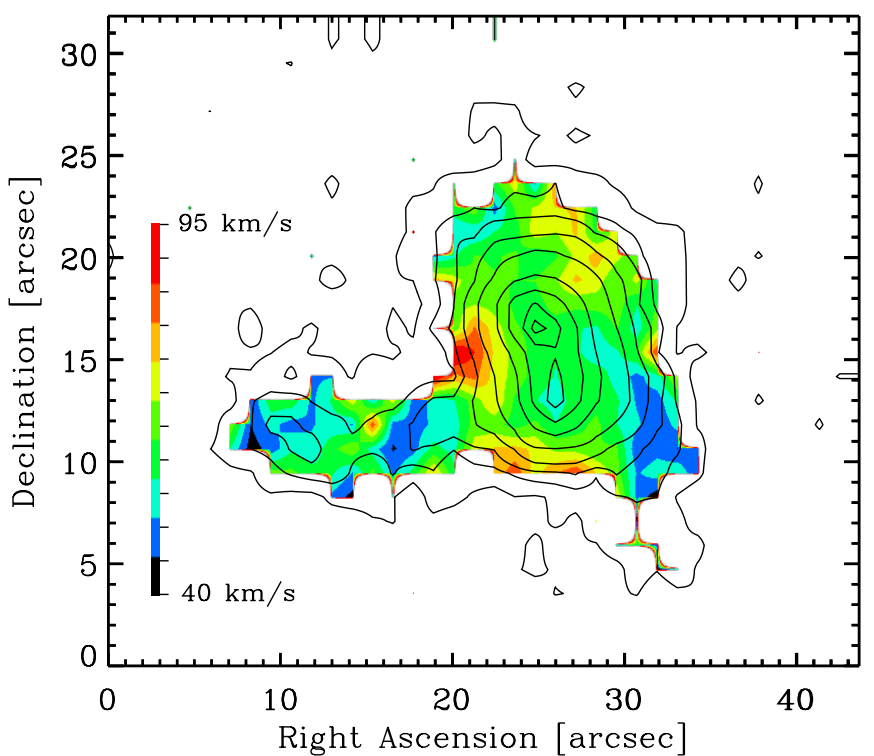

Fig. 3. The velocity dispersion map of galaxies $\mathrm{A}$ and $\mathrm{C}$ in $\mathrm{H} \alpha$. The colour scale shows the range in velocity dispersion values. Here, we characterize the velocity dispersion as the standard deviation of the Gaussian fit to the velocity profile. Only galaxies A and C showed significant internal variations in velocity dispersion, so they are the only ones shown here. The contours show the total $\mathrm{H} \alpha$ flux from the Fabry-Perot observations. As usual, north is up, east is to the left, and $1^{\prime \prime}=281 \mathrm{pc}$.

dispersions in galaxies $\mathrm{B}, \mathrm{E}$, and $\mathrm{F}$ are approximately $50 \mathrm{~km} \mathrm{~s}^{-1}$, similar to our instrumental resolution, while the velocity dispersion in galaxy $\mathrm{G}$ is slightly higher.

Table 1 lists the raw line intensities relative to $\mathrm{H} \beta$ and the raw radial velocities we measured in the $\mathrm{H}_{\text {II }}$ regions in the different components of HCG 31. The uncertainties quoted for the line intensities are $1 \sigma$ uncertainties and include the uncertainty in the fits to both the line and the reference line as well as the uncertainty in the continuum placement for both lines. The line intensities are not corrected for reddening. The names coincide with those indicated in Fig. 1. Table 1 also includes the reddening derived from the Balmer lines along with the abundance of doubly ionized oxygen and an estimate of the total oxygen abundance for those cases when we could derive an electron temperature.

Before computing the reddenings listed in Table 1, we corrected the Balmer lines for underlying absorption according to

$F_{\mathrm{c}}(\lambda)=F_{\mathrm{o}}(\lambda) \times\left(1+\frac{W_{\mathrm{abs}}}{W_{\lambda}}\right) /\left(1+\frac{W_{\mathrm{abs}}}{W_{\mathrm{H} \beta}}\right)$,

where $F_{\mathrm{o}}(\lambda)$ and $F_{\mathrm{c}}(\lambda)$ are the observed and corrected fluxes and $W_{\mathrm{abs}}, W_{\lambda}$, and $W_{\mathrm{H} \beta}$ are the equivalent widths of the underlying stellar absorption, of the emission line in question, and of $\mathrm{H} \beta$, respectively. We used $W_{\mathrm{abs}}=1.9 \AA$ (McCall et al. 1985) since our spectra had neither the resolution nor the signal-tonoise required to measure this directly. These corrected line intensities were then used to compute the reddening via

$\log \frac{F(\lambda)}{F(\mathrm{H} \beta)}=\log \frac{I(\lambda)}{I(\mathrm{H} \beta)}-0.4 E(B-V)\left(A_{1}(\lambda)-A_{1}(\mathrm{H} \beta)\right)$, 
Table 1. Raw line intensities ${ }^{a}$ for the $\mathrm{H}_{\text {II }}$ regions in HCG 31 measured from the multi-object spectroscopy.

\begin{tabular}{|c|c|c|c|c|c|c|c|c|}
\hline \multicolumn{2}{|l|}{$\overline{\lambda \lambda}$} & $\overline{\mathrm{A} 1+\mathrm{C} 1}$ & $\overline{\mathrm{C} 2}$ & $\overline{\mathrm{B} 2}$ & $\overline{\mathrm{B} 3}$ & $\overline{\mathrm{F} 1}$ & $\overline{\mathrm{F} 2}$ & $\overline{\mathrm{Q}}$ \\
\hline $\mathrm{H} \epsilon$ & 3970 & & $12.51 \pm 0.80$ & & & & & \\
\hline $\mathrm{H} \delta$ & 4101 & $10.44 \pm 0.99$ & $18.42 \pm 0.86$ & & & $10.3 \pm 2.7$ & & \\
\hline $\mathrm{H} \gamma$ & 4340 & $29.2 \pm 1.3$ & $38.4 \pm 1.1$ & & $33.6 \pm 3.3$ & $31.1 \pm 3.1$ & $31.9 \pm 5.3$ & \\
\hline [O III $]$ & 4363 & $2.20 \pm 0.93$ & $1.21 \pm 0.61$ & & & $5.1 \pm 2.5$ & & \\
\hline $\mathrm{He}_{\mathrm{I}}$ & 4472 & $1.14 \pm 0.97$ & $2.70 \pm 0.66$ & & & & & \\
\hline $\mathrm{H} \beta$ & 4861 & $100.0 \pm 2.8$ & $100.0 \pm 2.9$ & $100 \pm 16$ & $100.0 \pm 3.5$ & $100.0 \pm 2.6$ & $100.0 \pm 4.5$ & $100 \pm 32$ \\
\hline [O III] & 4959 & $71.6 \pm 3.2$ & $90.0 \pm 3.9$ & $135 \pm 19$ & $117.2 \pm 4.6$ & $171.8 \pm 6.3$ & $142.9 \pm 6.4$ & \\
\hline [O III] & 5007 & $218.1 \pm 5.4$ & $273.5 \pm 6.9$ & $406 \pm 47$ & $361.3 \pm 9.8$ & $521 \pm 12$ & $429 \pm 15$ & $157 \pm 43$ \\
\hline $\mathrm{He}_{\mathrm{I}}$ & 5876 & $15.68 \pm 0.43$ & $11.67 \pm 0.42$ & & $12.0 \pm 1.3$ & $13.83 \pm 0.91$ & $13.8 \pm 1.6$ & \\
\hline$\left[\mathrm{O}_{\mathrm{I}}\right]$ & 6300 & $8.77 \pm 0.42$ & $4.77 \pm 0.39$ & & $10.7 \pm 1.3$ & $8.06 \pm 0.93$ & & \\
\hline [O I $]$ & 6364 & $2.39 \pm 0.28$ & $1.30 \pm 0.13$ & & $1.51 \pm 0.92$ & $2.42 \pm 0.67$ & & \\
\hline $\mathrm{H} \alpha$ & 6563 & $473 \pm 10$ & $326.4 \pm 7.2$ & $625 \pm 71$ & & $367.4 \pm 7.1$ & $385 \pm 13$ & $470 \pm 109$ \\
\hline$\left[\mathrm{N}_{\text {II }}\right]$ & 6583 & $74.6 \pm 3.9$ & $34.1 \pm 2.4$ & $57 \pm 12$ & & $11.8 \pm 1.7$ & $12.9 \pm 2.5$ & $51 \pm 20$ \\
\hline $\mathrm{He}_{\mathrm{I}}$ & 6678 & $5.4 \pm 1.0$ & $3.55 \pm 0.34$ & & & $5.23 \pm 0.88$ & & \\
\hline$\left[\mathrm{S}_{\mathrm{II}}\right]$ & 6716 & $47.8 \pm 2.1$ & $25.01 \pm 0.98$ & & & $24.8 \pm 1.9$ & $23.6 \pm 2.5$ & $146 \pm 37$ \\
\hline$\left[\mathrm{S}_{\mathrm{II}}\right]$ & 6731 & $38.7 \pm 1.8$ & $17.54 \pm 0.77$ & & & $14.6 \pm 1.7$ & $9.3 \pm 2.4$ & $125 \pm 33$ \\
\hline$V_{\mathrm{r}}(\mathrm{H} \alpha)$ & $\left.\mathrm{m} \mathrm{s}^{-1}\right)$ & 3879 & 3947 & 4068 & & 3843 & 3831 & 4148 \\
\hline$E(B-$ & (mag) & $0.42 \pm 0.02$ & $0.10 \pm 0.02$ & $0.42 \pm 0.10$ & $0.68 \pm 0.44$ & $0.21 \pm 0.02$ & $0.26 \pm 0.03$ & $0.11 \pm 0.22$ \\
\hline$T_{\mathrm{e}}\left(\left[\mathrm{O}_{\mathrm{I}}\right.\right.$ & $(\mathrm{K})$ & $12500 \pm 3200$ & $9100 \pm 2000$ & & & $11900 \pm 3200$ & & \\
\hline $12+10$ & $\left.D^{++} / \mathrm{H}\right)$ & $7.56 \pm 0.34$ & $8.12 \pm 0.39$ & & & $8.02 \pm 0.38$ & & \\
\hline $12+10$ & $/ \mathrm{H})_{T \mathrm{e}}$ & $8.1 \pm 0.2$ & $8.3 \pm 0.2$ & & & $8.1 \pm 0.2$ & & \\
\hline
\end{tabular}

a The line intensities are measured relative to $\mathrm{H} \beta$ and are not corrected for interstellar reddening.

where $F(\lambda)$ and $I(\lambda)$ are the observed and theoretical emissionline fluxes at wavelength $\lambda, E(B-V)$ is the reddening, and $A_{1}(\lambda)=A(\lambda) / E(B-V)$. Values of $A_{1}(\lambda)$ were taken from the Schild (1977) reddening law. We used the $\mathrm{H} \alpha / \mathrm{H} \beta$ ratio to determine the reddening, except when $\mathrm{H} \alpha$ fell outside our wavelength range, in which case the $\mathrm{H} \gamma / \mathrm{H} \beta$ ratio was used. In all cases, we adopted theoretical ratios of $I(\mathrm{H} \alpha) / I(\mathrm{H} \beta)=$ 2.86 and $I(\mathrm{H} \gamma) / I(\mathrm{H} \beta)=0.468$, appropriate for electron temperatures and densities of $10^{4} \mathrm{~K}$ and $100 \mathrm{~cm}^{-3}$, respectively (Osterbrock 1989).

The radial velocities given in Table 1 are the raw radial velocities measured for the $\mathrm{H} \alpha$ line from the multi-object spectra, and are meant primarily to show that galaxy $\mathrm{Q}$ has a radial velocity consistent with group membership, and similar to the H I velocity found by Williams et al. (1991). Galaxy Q is the faintest of the components we observed, which may explain why it is so faint in the $\mathrm{H} \alpha$ image presented by Rubin et al. (1990) and absent from our $\mathrm{H} \alpha$ data cubes. We cannot restrict galaxy Q's morphological type since the $V$-band and $\mathrm{H} \alpha$ images we obtained to identify $\mathrm{H}$ II regions are of low spatial resolution and signal-to-noise and were never calibrated.

The abundance of doubly ionized oxygen is computed according to the prescription given in Richer (1993) using the reddening-corrected line intensities and assuming an electron density of $100 \mathrm{~cm}^{-3}$. The total oxygen abundances are estimated from Fig. 5 of Kobulnicky et al. (1999) in those cases when the electron temperature was measured. Generally, these total oxygen abundances are in good agreement with extant data (see Table 2).

In Table 2, we list the luminosities and oxygen abundances for the component galaxies with measured oxygen abundances.
Table 2. Luminosities and oxygen abundances.

\begin{tabular}{lccll}
\hline \hline Galaxy & $B$ & $12+\log (\mathrm{O} / \mathrm{H})$ & method $^{\mathrm{b}}$ & ref. \\
\hline A & $15.62 \pm 0.5$ & $8.04 \pm 0.06$ & $\mathrm{~A}$ & 1,3 \\
$\mathrm{~B}$ & $15.35 \pm 0.2$ & $8.34 \pm 0.20$ & $\mathrm{~B}$ & 1,4 \\
$\mathrm{C}$ & $13.44 \pm 0.5$ & $8.3 \pm 0.2$ & $\mathrm{~A}, \mathrm{C}$ & $1,5,6$ \\
$\mathrm{~F}$ & $18.2 \pm 1.0^{\mathrm{a}}$ & $8.1 \pm 0.2$ & $\mathrm{C}$ & 2,6 \\
\hline
\end{tabular}

a The luminosity of component $\mathrm{F}$ is assumed equal to that of component E.

b The oxygen abundances are obtained by the following methods: (A) measurements of [O II] and [O III] lines and the electron temperature; (B) measurements of [O II] and [O III] lines only; (C) measurement of the $[\mathrm{O}$ III] lines and the electron temperature.

References: (1) Hickson et al. (1988); (2) Iglesias-Páramo \& Vílchez (1997); (3) Izotov \& Thuan (1998); (4) Rubin et al. (1990); (5) Vacca \& Conti (1992); (6) Table 1.

For each galaxy, we adopted what appeared to be the best abundance determination available, indicated in the footnotes to Table 2, using the precision quoted in the original papers as our guide. For galaxies A, B, and C, we adopted the Hickson et al. (1988) $B$-band magnitudes at the $24.5 \mathrm{mag} / \square^{\prime \prime}$ isophote since these seem to be in better agreement with the photometry of Iglesias-Páramo \& Vílchez (1997). No photometry exists for galaxy F. Here, we assume it has the same luminosity as galaxy E (Iglesias-Páramo \& Vílchez 1997), which, though it is a rough approximation, is sufficient for our purposes. In Table 2, we adopted the uncertainties in the luminosities of galaxies B and C from Hickson et al. (1988). For galaxy A, we set the uncertainty in luminosity equal to that for galaxy $\mathrm{C}$ 

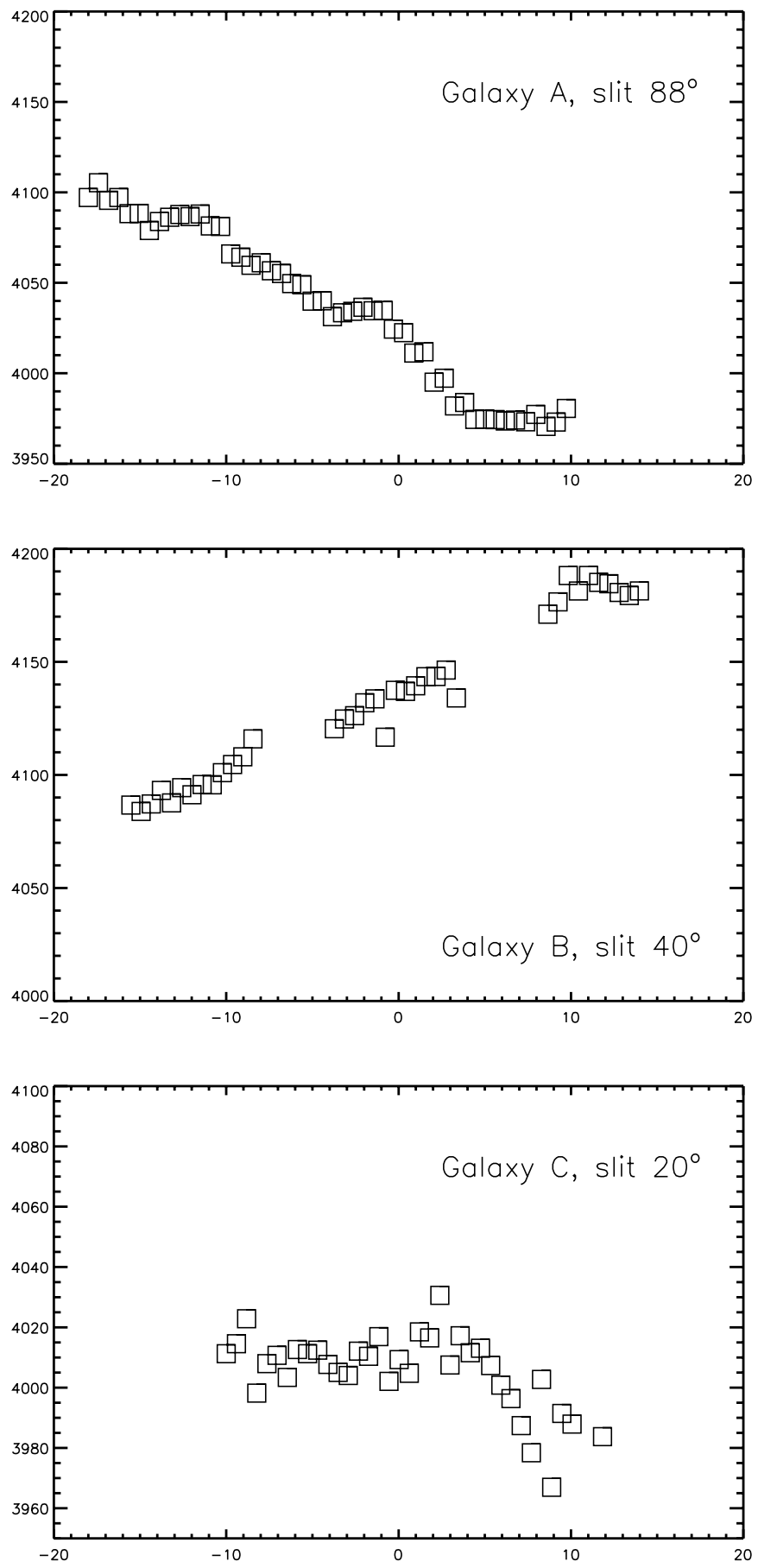

Fig. 4. This figure presents velocity profiles of galaxies A, B, and C extracted at the same positions and position angles as Rubin et al. (1990). Each panel indicates the position angle of the simulated slit. The agreement is very good for both the velocities and the velocity profiles, any discrepancies being of order $10 \mathrm{~km} \mathrm{~s}^{-1}$ (compare with their Figs. 2 and 3). We are not as sensitive to low surface brightness emission, so our profiles have a smaller spatial extent than those of Rubin et al. (1990).

due to the difficulty of disentangling the two galaxies. We arbitrarily estimate an uncertainty of $1.0 \mathrm{mag}$ in the luminosity of galaxy F. In Fig. 5, we plot the oxygen abundance as a function of the galaxy luminosity. We converted from apparent to absolute magnitudes using an apparent distance modulus of

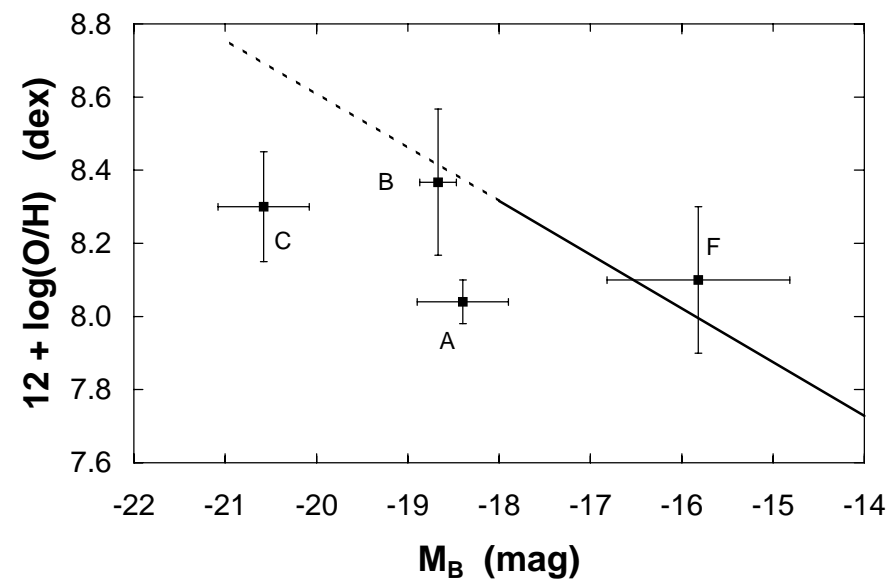

Fig. 5. The relation between luminosity and metallicity for the galaxies in HCG 31. The components are labelled. The solid line is the relation for dwarf irregulars found by Richer \& McCall (1995), while the dashed line is an extrapolation of that relation. The galaxies in HCG 31 are clearly discrepant, with components A and C much too luminous for their abundances.

$34.02 \mathrm{mag}$, which accounts for our adopted distance and an extinction of $A_{B}=0.21 \mathrm{mag}$, based upon $E(B-V)=0.055 \mathrm{mag}$ (Schlegel et al. 1998) and the Fitzpatrick (1999) reddening law $\left(R_{V}=3.1\right.$ and $\left.E(B-V)=1 \mathrm{mag}\right)$. We also show the relation between oxygen abundance and luminosity for dwarf irregulars for comparison (Richer \& McCall 1995).

\section{The state of HCG 31 today}

Our most surprising result is that galaxies $\mathrm{A}$ and $\mathrm{C}$ appear to be a single kinematical entity. In Fig. 2, their kinematics show no discontinuity. We interpret this as evidence that galaxies A and $\mathrm{C}$ are a single entity at present. Mendes de Oliveira \& Amram (2000) and Amram \& Mendes de Oliveira (2000) find similar results from similar kinematical data.

Another surprise is that galaxy E's position and velocity are compatible with its also being an integral part of the $\mathrm{A}+\mathrm{C}$ complex. If galaxy $\mathrm{E}$ is in fact a separate entity, it is just now separating from the $\mathrm{A}+\mathrm{C}$ complex. In any case, its kinematics confirm that the extension of the $\mathrm{A}+\mathrm{C}$ complex towards galaxies $\mathrm{E}$ and $\mathrm{F}$ is a tidal tail.

Like galaxy E, the tidal dwarfs to the north-east of galaxies A and C identified by Hunsberger et al. (1996) also have kinematics consistent with their being integral components of the $\mathrm{A}+\mathrm{C}$ complex. Furthermore, given the red continuum image presented by Johnson \& Conti (2000), it is not evident that at least the nearer of these supposed tidal dwarfs are separate entities.

Meanwhile, galaxy $\mathrm{F}$ is an excellent example of a tidal fragment. Considering its position and radial velocity (Figs. 1 and 2), it seems clear that galaxy $F$ is a tidal fragment of the $\mathrm{A}+\mathrm{C}$ complex that detached some time ago. Remarkably, galaxy $\mathrm{F}$ has the same oxygen abundance as galaxies $\mathrm{A}$ and $\mathrm{C}$, despite being more than 5 mag fainter than galaxy $\mathrm{C}$ (cf. Table 2 and Fig. 5). In addition, since an underlying stellar population has yet to be detected in galaxy F (Johnson \& Conti 2000), it cannot be responsible for its own chemical 
enrichment. All of these observations argue that galaxy $\mathrm{F}$ is a tidal fragment of the $\mathrm{A}+\mathrm{C}$ complex.

Galaxy B exhibits solid-body rotation. Given its broadband morphology (Rubin et al. 1990; Iglesias-Páramo \& Vílchez 1997), it is likely a dwarf spiral or irregular seen nearly edgeon. Kinematically, galaxy B is distinct from the $\mathrm{A}+\mathrm{C}$ complex and is counter-rotating with respect to it.

Galaxy $G$ appears to be a small disk galaxy seen nearly face-on since the $\mathrm{H} \alpha$ velocity has only a very small gradient across its disk.

The velocity dispersion map (Fig. 3) of the ionized gas is more difficult to interpret. The highest velocity dispersions occur along a ridge between the main bodies of galaxies $\mathrm{A}$ and $\mathrm{C}$. Whether this higher velocity dispersion is due to a more complicated gas distribution or kinematics is indeterminate from our observations.

It is evident from Fig. 5 that the oxygen abundances in the galaxies in HCG 31 are unusual. Although they span 5 mag in luminosity, all of the galaxies have nearly identical oxygen abundances. This conclusion is independent of the uncertainty in separating the luminosities of galaxies A and C, since, were we to consider their combined luminosity and either of the oxygen abundances observed (Table 2), the resulting position would remain highly discrepant compared to the relation for normal dwarf irregular galaxies. However, galaxy C's luminosity is much greater than that of most dwarfs and more like that of a typical late-type spiral. If the $\mathrm{A}+\mathrm{C}$ complex were a late-type spiral, its oxygen abundance may be more easily understood. Given the strong inflows and consequent high star formation rate expected due to tidal interactions (e.g., Mihos \& Hernquist 1996; Barnes \& Hernquist 1996), the mass of gas expected in the outer disks of late-type spirals, and the low oxygen abundances observed in these regions (Ferguson et al. 1998; van Zee et al. 1998), the unusual positions of galaxies A and $\mathrm{C}$ in Fig. 5 are understandable as the result of dilution due to strong gas inflow and the transient high luminosity due to a starburst. At any rate, galaxy C's position is compatible with the extreme low end of the abundance range for late-type spirals (Garnett et al. 1997). Given galaxy F's origin within the $\mathrm{A}+\mathrm{C}$ complex, it should naturally have the same oxygen abundance as the $\mathrm{A}+\mathrm{C}$ complex.

Galaxy B, meanwhile, appears more or less normal. It has a $B$-band luminosity at the bright end of the range found for dwarf irregulars, about 0.8 mag brighter than the LMC's, and an oxygen abundance similar to that of the LMC. Its $\mathrm{H} \alpha$ luminosity, $1.0 \times 10^{41} \mathrm{erg} \mathrm{s}^{-1}$ (Iglesias-Páramo \& Vílchez 1997 , corrected to our adopted distance), implies a global star formation rate of $0.8 M_{\odot} \mathrm{yr}^{-1}$ (Kennicutt 1998), which is not unusual for a late-type galaxy (Kennicutt 1983). However, its colours are significantly bluer than those of typical dwarf irregulars or late-type spirals (Iglesias-Páramo \& Vílchez 1997; de Vaucouleurs et al. 1981; Roberts \& Haynes 1994), being a near-perfect match to those of a single stellar population of metallicity $Z=0.008$ and an age of $40 \mathrm{Myr}$ (Bressan et al. 1994). Thus, galaxy B is currently forming stars more actively than it has formed them on average in the past, perhaps as a result of the group environment. In spite of this, galaxy B's recent history of star formation appears to be distinct from that of the A+C complex (Iglesias-Páramo \& Vílchez 1997; Johnson \& Conti 2000).

\section{The past and future}

The kinematical evidence argues strongly that the A+C complex has undergone very significant, recent, tidal interaction, but that it is now a single entity. In addition, the kinematical properties of galaxies E, F, and the tidal candidates identified by Hunsberger et al. (1996) all provide evidence of their origin within the $\mathrm{A}+\mathrm{C}$ complex. What, then, are the possible origins of these tidal interactions, and might these shed some light on the nature of HCG 31? A related issue is that of the nature of the original components of HCG 31 .

The global H I mass and blue luminosity of HCG 31 provide some clues regarding the original composition of HCG 31 . The $\mathrm{H}_{\mathrm{I}}$ mass of HCG 31 is strikingly high, $2.5 \times 10^{10} M_{\odot}$ adjusted to our adopted distance (Williams et al. 1991), which would be typical of a massive Sb or Sc spiral (Roberts \& Haynes 1994). The oxygen abundances, however, are incompatible with those of such massive galaxies save those found in their extreme outer disks (Garnett et al. 1997; Ferguson et al. 1998; van Zee et al. 1998). The blue luminosities of galaxies $A+C$, B, and G imply morphological types Scd, Sm, and Sd (Roberts \& Haynes 1994), though these are all undoubtedly affected by their significant recent star formation. The total H I mass is also consistent with the sum of three late-type spirals (or one such spiral and two bright irregulars; Roberts \& Haynes 1994). As noted above, the oxygen abundances of $\mathrm{A}+\mathrm{C}$ and $\mathrm{B}$ are compatible with such progenitors, though that for $\mathrm{A}+\mathrm{C}$ would be at the low end of the range expected (Richer \& McCall 1995; Garnett et al. 1997). Finally, the global $L_{B} / M(\mathrm{H} \mathrm{I})$ for the entire group is approximately unity, a value typical of dwarf irregulars (Roberts \& Haynes 1994). Despite the uncertainty due to the recent star formation, all of the available evidence points toward the group being initially composed of late-type spirals and irregulars.

The kinematical evolution of HCG 31 is a more ambiguous puzzle. Based upon the galaxy morphologies and less complete kinematical data than we present here, an on-going merger between galaxies $\mathrm{A}$ and $\mathrm{C}$ has been invoked to explain the tidal features observed (e.g., Rubin et al. 1990). However, this explanation leaves a number of issues unresolved, the two most important of which are the coincidence of galaxy $G$ with a peak in the $\mathrm{H}_{\mathrm{I}}$ distribution at the end of a tidal tail and the very similar recent star formation histories in galaxies $\mathrm{A}, \mathrm{C}$, E, F, and G (Williams et al. 1991; Iglesias-Páramo \& Vílchez 1997; Johnson et al. 1999). The continuity of the kinematics of the $\mathrm{A}+\mathrm{C}$ complex is an additional important complication since it implies that the interaction would be in an advanced, settled stage, in contrast to numerical simulations that indicate that most of the star formation ought to occur at the onset of the interaction (Barnes \& Hernquist 1996; Mihos \& Hernquist 1996). Likewise, the blue colours of the star clusters in galaxies $\mathrm{A}$ and $\mathrm{C}$ imply that any merger should be in an initial phase (Johnson et al. 1999).

In view of the continuous kinematics of the $A+C$ complex (see also Amram \& Mendes de Oliveira 2000), we propose that 
an encounter between it and galaxy $\mathrm{G}$ is a more likely origin for the tidal interaction that is so evident. This encounter scenario naturally accounts for the position of galaxy $\mathrm{G}$, embedded in the south-east tidal tail of the $\mathrm{H}_{\mathrm{I}}$ distribution (Williams et al. 1991). An encounter between galaxy $G$ and the $A+C$ complex also easily explains the very similar histories of the most recent star formation in galaxies $\mathrm{A}+\mathrm{C}, \mathrm{E}, \mathrm{F}$, and $\mathrm{G}$ noted by Iglesias-Páramo \& Vílchez (1997) and Johnson \& Conti (2000), despite their physical separation of up to $40 \mathrm{kpc}$, since such an encounter would provoke strong gas inflow and presumably vigourous star formation in galaxy $\mathrm{G}$, the $\mathrm{A}+\mathrm{C}$ complex, and in the tidal material (Mihos et al. 1992; Mihos \& Hernquist 1996; Barnes \& Hernquist 1996). Such strong gas inflow would also explain the low oxygen abundance measured in the $\mathrm{A}+\mathrm{C}$ complex as a result of dilution by metal-poor gas. The coordinated star formation, the global morphology and kinematics of the H I gas (Williams et al. 1991), and the positions of galaxies $\mathrm{A}+\mathrm{C}, \mathrm{E}, \mathrm{F}$, and $\mathrm{G}$ are all very reminiscent of the fly-by and merger simulations of Mihos et al. (1992), Mihos \& Hernquist (1996), and Barnes \& Hernquist (1996). Finally, O'Halloran et al. (2002) conclude that the burst of star formation in the $\mathrm{A}+\mathrm{C}$ complex may be evolving to a post-starburst phase, based upon ISO data, a result compatible with the encounter scenario if galaxy $\mathrm{G}$ is now moving away from the $\mathrm{A}+\mathrm{C}$ complex. Therefore, unless galaxy $\mathrm{G}$ occupies its position purely by chance, we propose that an encounter between it and the $\mathrm{A}+\mathrm{C}$ complex accounts for more of the properties of HCG 31 in a more natural fashion than does a merger of galaxies $\mathrm{A}$ and $\mathrm{C}$.

An important uncertainty in the encounter scenario is the transverse velocity implied between galaxy $\mathrm{G}$ and the $\mathrm{A}+\mathrm{C}$ complex. Given their very similar radial velocities, the bulk of their relative motion must be in the plane of the sky (Fig. 2; Rubin et al. 1990; Hickson et al. 1992). At a distance of $58 \mathrm{Mpc}$, the 2!4 separation of galaxy $\mathrm{G}$ and the $\mathrm{A}+\mathrm{C}$ complex corresponds to a linear distance of $40 \mathrm{kpc}$. If the ages of the most recent star formation, $<10^{8}$ years (Iglesias-Páramo \& Vílchez 1997; Johnson et al. 1999), are taken as the time elapsed since the putative encounter, the separation implies a transverse velocity in the plane of the sky exceeding $400 \mathrm{~km} \mathrm{~s}^{-1}$. This velocity greatly exceeds the internal velocity dispersions we measure in these galaxies (Fig. 3; Sect. 3), and it is not obvious whether this encounter velocity should provoke such a strong interaction (e.g., Binney \& Tremaine 1987). The time scale could be lengthened, and the transverse velocity lowered, if there is a delay between closest approach and the initiation of star formation, as some models indicate (e.g., Mihos et al. 1992; Mihos \& Hernquist 1996). On the other hand, neither the velocity nor the spatial extension between galaxy $\mathrm{G}$ and the $\mathrm{A}+\mathrm{C}$ complex are unusual. HCG 26 is very similar (Williams \& van Gorkom 1995), while HCG 16 has a similar morphology on a larger scale (Verdes-Montenegro et al. 2001). NGC 7318b in Stephan's Quintet (HCG 92) has been stripped of its $\mathrm{HI}_{\mathrm{I}}$ despite a velocity of order $1000 \mathrm{~km} \mathrm{~s}^{-1}$ relative to the other group members (Sulentic et al. 2001). Likewise, UGC 7636 in the Virgo cluster has had its interstellar medium stripped by NGC 4472 though their relative velocities differ by about $700 \mathrm{~km} \mathrm{~s}^{-1}$ (Patterson \& Thuan 1992; McNamara et al. 1994; Lee et al. 2000).Therefore, although the response of the $\mathrm{H}_{\mathrm{I}}$ in fly-by encounters appears to be relatively unexplored theoretically, observational examples appear reasonably common, lending credence to the idea that an interaction between galaxies $\mathrm{G}$ and $\mathrm{A}+\mathrm{C}$ could be the origin of the tidal debris seen in HCG 31.

If the gravitational interaction between galaxy $\mathrm{G}$ and the $\mathrm{A}+\mathrm{C}$ complex is the origin of the tidal debris in HCG 31, it is then also possible that the $\mathrm{A}+\mathrm{C}$ complex has always been a single entity and need not represent the fusion of two galaxies, partially confirming the hypothesis of Plana et al. (2000). The simulations of fly-by and merger interactions indicate that very strong gas inflows may be induced (Mihos et al. 1992; Mihos \& Hernquist 1996; Barnes \& Hernquist 1996) and such inflows are indeed observed in HCG 31 (Williams et al. 1991), though the $\mathrm{H}_{\mathrm{I}}$ kinematics are slightly compromised since their spatial resolution is insufficient to clearly separate the gas belonging to galaxy $\mathrm{B}$ from that belonging to the $\mathrm{A}+\mathrm{C}$ complex. The distribution of the star formation observed in the $\mathrm{A}+\mathrm{C}$ complex could simply be a typical result of the gas inflows predicted by the numerical simulations. Likewise, the low oxygen abundances could easily result from dilution due to the inflow of metal-poor material from the outer disk.

What might be the eventual outcome of HCG 31? If an encounter between galaxy $\mathrm{G}$ and the $\mathrm{A}+\mathrm{C}$ complex is the origin of the tidal interactions, and if their orbits are unbound, the $\mathrm{A}+\mathrm{C}$ complex will likely evolve similar to the simulations of ring galaxies (e.g., Struck 1997), and the A+C complex should remain as a disk galaxy. If, on the contrary, their orbits are bound, a merger that will likely also consume galaxies E, F, and the tidal dwarfs postulated by Hunsberger et al. (1996) is the probable result. Finally, if the interaction between galaxies A and C is the origin of the tidal debris in HCG 31, as has been usually supposed (e.g., Rubin et al. 1990; Johnson et al. 1999; Mendes de Oliveira \& Amram 2000), a merger already appears to be well underway.

In either case of a merger, we expect that a low mass elliptical would be the eventual outcome, as suggested by Rubin et al. (1990). The group's H $\alpha$ luminosity is $1 \times 10^{42} \mathrm{erg} \mathrm{s}^{-1}$ (Iglesias-Páramo \& Vílchez 1997, adjusted to our distance), implying a star formation rate of $7.9 M_{\odot} \mathrm{yr}^{-1}$ (Kennicutt 1998). Although high, this rate of star formation is sustainable for a long time, at least another 3 Gyr given the group's Hi mass of $2.5 \times 10^{10} M_{\odot}$ (Williams et al. 1991). Most of this H I mass is contained within an area of $3^{\prime} \times 1^{\prime}$ (Williams et al. 1991), $8.6 \times 10^{8} \mathrm{pc}^{2}$ at our adopted distance, implying a gas surface mass density of about $30 M_{\odot} \mathrm{pc}^{-2}$. Supposing a conversion efficiency from Hi gas into stars of approximately $10 \%$ at this surface density (Kennicutt 1998), and also supposing that at least $50-60 \%$ of the mass converted to stars will produce longlived stars (Köppen \& Arimoto 1991), we infer that the eventual outcome could be a galaxy with a stellar mass of several $10^{9} M_{\odot}$. This mass is several times larger than the current mass estimates: the colours from Iglesias-Páramo \& Vílchez (1997) are completely dominated by the light from young stars, and therefore imply only lower limits to the stellar mass of order $3 \times 10^{8} M_{\odot}$, based upon the colours of the single stellar 
populations from Bressan et al. (1994). Therefore, it is reasonable to expect an eventual metal production at least equal to that which has already occurred, accompanied by a similar increase in the chemical abundances. Several Gyr after the cessation of star formation, a galaxy with a stellar mass of several $10^{9} M_{\odot}$ would have a luminosity of $M_{B} \approx-18 \mathrm{mag}$ (e.g., Bressan et al. 1994) and its oxygen abundance would place it above the metallicity-luminosity relation for dwarf irregulars. Given that the velocity gradients in the $\mathrm{H}_{\mathrm{I}}$ gas found by Williams et al. (1991) imply generally radial inflow, rather than rotation, we infer that the probable outcome will be an elliptical galaxy. Although the foregoing is entirely speculative, it illustrates that HCG 31 could very plausibly produce a typical low-luminosity elliptical with normal chemical abundances (e.g., Richer et al. 1998).

\section{Conclusions}

Somewhat at variance with previous studies, it is less evident to us that HCG 31 is a clear example of an on-going merger. It is undeniable, however, that there is significant tidal interaction underway among the members of this compact group. Given their kinematics, galaxies A, C, and the tidal dwarfs to the north-east identified by Hunsberger et al. (1996) all appear to be a single entity at present (the $\mathrm{A}+\mathrm{C}$ complex). Galaxy $\mathrm{E}$ is probably also an integral part of this complex, otherwise it is a tidal fragment that has very recently separated. Galaxy F has both kinematics and chemical abundances that indicate that it is a tidal fragment that was part of the $\mathrm{A}+\mathrm{C}$ complex in the relatively recent past. At this point, galaxies $B$ and $G$ remain kinematically distinct from the $\mathrm{A}+\mathrm{C}$ complex. Finally, we find a velocity for galaxy Q (Rubin et al. 1990) that is compatible with it also being a member of HCG 31 .

We propose that a gravitational interaction between galaxy $\mathrm{G}$ and the $\mathrm{A}+\mathrm{C}$ complex is the most complete explanation of the events occurring in HCG 31 . This scenario naturally accounts for the positions and kinematics of galaxies A, C, E, F, $\mathrm{G}$, and the tidal dwarf candidates of Hunsberger et al. (1996), the morphology and kinematics of the $\mathrm{H}_{\mathrm{I}}$ (Williams et al. 1991), the chemical abundances, and the coordinated star formation in galaxies separated by up to $40 \mathrm{kpc}$ (Iglesias-Páramo \& Vílchez 1997; Johnson \& Conti 2000). In this case, the A+C complex may have always been a single entity, a late-type spiral, and its current irregular morphology, high luminosity, blue colours, and anomalous chemical abundance may simply be a consequence of multiple bursts of star formation as a result of strong gas inflows induced by its interaction with galaxy $\mathrm{G}$ (Barnes \& Hernquist 1996; Mihos \& Hernquist 1996). If this is the case, a merger may still eventually occur between galaxy $\mathrm{G}$ and the $\mathrm{A}+\mathrm{C}$ complex given the ample evidence for a strong gravitational interaction. While there is no evidence that the kinematical properties of HCG 31 are not the result of an ongoing merger between galaxies $\mathrm{A}$ and $\mathrm{C}$, many additional details are not accounted for by this solution, particularly the coordinated star formation and the location of galaxy $\mathrm{G}$.

Based upon its oxygen abundance, its broad-band and $\mathrm{H} \alpha$ luminosities, and its kinematics, at present galaxy $\mathrm{B}$ appears to be a relatively normal late-type spiral or bright dwarf irregular seen nearly edge-on. Its evolution is probably now being affected by the group environment, since its colours are very blue and indicate that its current rate of star formation exceeds its historical average rate.

Acknowledgements. MGR thanks Jack Sulentic, Héctor Aceves, and Héctor Velazquez for very helpful discussions. We also thank the referee, Philippe Amram, for careful and useful comments. MGR thanks the staff of the Observatorio Astrofísico Guillermo Haro for their hospitality and Raúl González and Gerardo Miramon for their able assistance with the observations. MR and LG thank Gabriel Garcia and Gustavo Melgoza for their assistance with the observations at the Observatorio Astronómico Nacional in San Pedro Mártir. MGR acknowledges financial support from DGAPA project IN100799 and CONACyT project 37214-E. MR, MGR, and AB acknowledge financial support from CONACyT project 27984-E and DGAPA project IN122298. LG acknowledges financial support from DGAPA project IN113999. AB acknowledges a Mutis graduate fellowship from the Agencia Española de Cooperación Internacional. MVG acknowledges a CONACyT scholarship (number 114735), and also thanks the time allocation committee at INAOE for assigning time to this project.

\section{References}

Amram, P., \& Mendes de Oliveira, C. 2000, in Dynamics of Galaxies: from the Early Universe to the Present, ASP Conf. Ser., 197, 369

Barnes, J. E., \& Hernquist, L. 1996, ApJ, 471, 115

Binney, J., \& Tremaine, S. 1987, Galactic dynamics (Princeton, NJ, Princeton University Press), 747

Bressan, A., Chiosi, C., \& Fagotto, F. 1994, ApJS, 94, 63

de Vaucouleurs, G., de Vaucouleurs, A., \& Buta, R. 1981, AJ, 86, 1429

Ferguson, A. M. N., Gallagher, J. S., \& Wyse, R. F. G. 1998, AJ, 116, 673

Fitzpatrick, E. L. 1999, PASP, 111, 63

Garnett, D. R., Shields, G. A., Skillman, E. D., Sagan, S. P., \& Dufour, R. J. 1997, ApJ, 489, 63

Hickson, P. 1982, ApJ, 255, 382

Hickson, P., Kindl, E., \& Huchra, J. P. 1988, ApJ, 331, 64

Hickson, P., Mendes de Oliveira, C., Huchra, J. P., \& Palumbo, G. G 1992, ApJ, 399, 353

Hunsberger, S. D., Charlton, J. C., \& Zaritsky, D. 1996, ApJ, 462, 50

Iglesias-Páramo, J., \& Vílchez, J. M. 1997, ApJ, 479, 190

Izotov, Y. I., \& Thuan, T. X. 1998, ApJ, 500, 188

Johnson, K. E., \& Conti, P. S. 2000, AJ, 119, 2146

Johnson, K. E., Vacca, W. D., Leitherer, C., Conti, P. S., \& Lipscy, S. J. 1999, AJ, 117, 1708

Kennicutt, R. C. 1983, ApJ, 272, 54

Kennicutt, R. C. 1998, ARA\&A, 36, 189

Kobulnicky, H. A., Kennicutt, R. C., \& Pizagno, J. L. 1999, ApJ, 514, 544

Köppen, J., \& Arimoto, N. 1991, A\&AS, 87, 109

Le Coarer, E., Rosado, M., Georgelin, Y., Viale, A., \& Goldes, G. 1993, A\&A, 280, 365

Lee, H., Richer, M. G., \& McCall, M. L. 2000, ApJ, 530, L17

McCall, M. L., Rybski, P. M., \& Shields, G. A. 1985, ApJS, 57, 1

McNamara, B. R., Sancisi, R., Henning, P. A., \& Junor, W. 1994, AJ, 108,844

Mendes de Oliveira, C., \& Amram, P. 2000, in Small Galaxy Groups, ASP Conf. Ser., 209, 60

Mihos, J. C., \& Hernquist, L. 1996, ApJ, 464, 641

Mihos, J. C., Richstone, D. O., \& Bothun, G. D. 1992, ApJ, 400, 153

Mould, J. R., Huchra, J. P., Freedman, W. L., et al. 2000, ApJ, 529, 786 
O'Halloran, B., Metcalfe, L., McBreen, B., et al. 2002, ApJ, 575, 747

Osterbrock, D. E. 1989, Astrophysics of gaseous nebulae and active galactic nuclei (Research supported by the University of California, John Simon Guggenheim Memorial Foundation, University of Minnesota, et al. Mill Valley, CA, University Science Books)

Patterson, R. J., \& Thuan, T. X. 1992, ApJ, 400, L55

Plana, H., Amram, P., Mendes de Oliveira, C., \& Balkowski, C. 2000, AJ, 120, 621

Richer, M. G. 1993, ApJ, 415, 240

Richer, M. G., \& McCall, M. L. 1995, ApJ, 445, 642

Richer, M. G., McCall, M. L., \& Stasinska, G. 1998, A\&A, 340, 67

Roberts, M. S., \& Haynes, M. P. 1994, ARA\&A, 32, 115

Rosado, M., Georgiev, L., Dultzin-Hacyan, D., \& Cruz-González, I. 1999, in Wolf-Rayet Phenomena in Massive Stars and Starburst Galaxies, IAU Symp., 193, 618

Rosado, M., Langarica, R., Bernal, A., et al. 1995, Rev. Mex. Astron. Astrof. Conf. Ser., 3, 263
Rubin, V. C., Hunter, D. A., \& Ford, W. K. J. 1990, ApJ, 365, 86 Schild, R. E. 1977, AJ, 82, 337

Schlegel, D. J., Finkbeiner, D. P., \& Davis, M. 1998, ApJ, 500, 525

Struck, C. 1997, ApJS, 113, 269

Sulentic, J. W., Rosado, M., Dultzin-Hacyan, D., et al. 2001, AJ, 122, 2993

Vacca, W. D., \& Conti, P. S. 1992, ApJ, 401, 543

van Zee, L., Salzer, J. J., Haynes, M. P., O’Donoghue, A. A., \& Balonek, T. J. 1998, AJ, 116, 2805

Verdes-Montenegro, L., Yun, M. S., Williams, B. A., et al. 2001, A\&A, 377, 812

Williams, B. A., McMahon, P. M., \& van Gorkom, J. H. 1991, AJ, 101,1957

Williams, B. A. \& van Gorkom, J. H. 1995, in Groups of Galaxies, ASP Conf. Ser., 70, 77

Zickgraf, F., Thiering, I., Krautter, J., et al. 1997, A\&AS, 123, 103 